Original article

\title{
Role of Contrast Enhanced Mammography in Assessment of Focal Breast Asymmetry
}

Ghada S. Eliwa ${ }^{\mathrm{a}}$, Hesham E. Elshaikh ${ }^{\mathrm{b}}$, Gamal E. Elhabbaa ${ }^{\mathrm{c}}$, Lamia A. Salah Eldin ${ }^{\mathrm{b}}$

\begin{abstract}
a Department of radiology, Mshtoul hospital ,Mashtoul, Sharkia, Egypt. ${ }^{\mathrm{b}}$ Department of radiology, Benha faculty of medicine, Banha University, Egypt. ${ }^{\mathrm{c}}$ Department of surgery, Benha faculty of medicine, Banha University, Egypt.
\end{abstract}

Correspondence to: Ghada S. Eliwa, Department of radiology, Mshtoul hospital ,Mashtoul, Sharkia, Egypt

Email:

ghada.salah.elsisi@gmail.com

Received: 2 May 2021

Accepted: 23 May 2021

\begin{abstract}
Background: Contrast enhanced mammography (CESM) is one of the relatively novel imaging modalities. Many studies have suggested that CESM is superior to mammography in the identification of multiplicity, extent and size of malignant lesions especially in the dense breast parenchyma Aim: Characterization of focal breast asymmetry by using intravenous contrast material with full-field digital mammography. Methods: This study included 60 female patients who presented for screening and diagnostic mammogram at mammography unit in Egyptian National Cancer Institute. All enrolled cases had focal breast asymmetries detected on mammogram. Ultrasound examination and CESM using dual-energy acquisitions were performed for all included patients. Histopathology after ultrasound guided true cut biopsy was the standard reference for detecting lesions Results: Regarding overall validity of CESM, it was
\end{abstract} found that CESM had sensitivity $92.85 \%$, specificity $88.9 \%$. PVP and PVN were $95.1 \%$ and $84.42 \%$ respectively with accuracy $91.66 \%$. CESM sensitivity increased to $93.9 \%$ in detection of mass enhanced lesions with accuracy 92.5\%. In non-mass enhanced CESM sensitivity decreased to $88.9 \%$ with accuracy $90 \%$ while its specificity was $90.9 \%$. In mass enhanced lesions shape of the mass had the highest sensitivity $90.0 \%$ and specificity $85.7 \%$ with accuracy $90.0 \%$. In nonmass lesions pattern of enhancement had the highest sensitivity $88.9 \%$. Conclusion: CESM is valid and reliable tool in assessment of focal asymmetries. Enhancement and morphology characteristics are valuable in differentiation of benign and malignant breast lesions.

Key words: Contrast Enhanced Mammography, focal asymmetry, validity 
List of Abbreviations:

(CC) Craniocaudal

(MLO) mediolateral oblique

(CAD) computer-aided detection

(DBT) digital breast tomosynthesis

(DCIS) ductal carcinoma in situ

(BI-RADS) Breast Imaging Reporting and Data System

(FDA) Food and Drug Administration

(MD) Mammographic density

\section{Introduction}

Breast cancer is a major health problem and a leading cause of death among women in Egypt. Early detection of breast cancer aims to reduce morbidity and mortality rates. Mammography has been established as the imaging modality for screening and early detection of breast cancer; however, it is accused of having low sensitivity and specificity in women with dense breasts. ${ }^{(1)}$

Accurate diagnosis and characterization of breast lesions have an essential role in the management and improved prognosis of breast cancer. ${ }^{(2)}$

Breast asymmetries can be quite normal and are often secondary to variations in normal breast tissue. Nevertheless, in some cases, they may indicate an underlying serious pathology. ${ }^{(3)}$ Breast asymmetries are
(IMLN) Intramammary lymph nodes

(US). Ultrasonography

(CEM) Contrast enhanced mammography

(SE) single-energy

(DE) dual-energy

(MRI) magnetic resonance imaging

(CESM) contrast-enhanced spectral mammography

(IDC) invasive ductal carcinoma

(BPE) Background parenchymal enhancement

(NME) non-mass enhancement

one of the most challenging mammographic findings to evaluate and they often entail further assessment with additional mammography views and a targeted ultrasound study. ${ }^{(4)}$

Focal asymmetry is defined when the same features are observable on standard mammographic views occupying less than a single quadrant, but lacking convex margins and containing interspersed fat. ${ }^{(5)}$ The incidence of asymmetric findings on mammograms varies, where focal asymmetry was reported in $0.87 \% .^{(6)}$

CESM is one of the relatively novel imaging modalities. It provides low energy images comparable to mammographic images and post- contrast recombined images to evaluate tumor neovascularity. 
CESM allows better evaluation of calcifications by their visualization on lowenergy images combined with enhancement criteria on the contrast enhanced recombined images. ${ }^{(7)}$

CEM is an alternative to US and MRI, and it can be used to obtain contrast material enhanced information and standard mammograms simultaneously. CEM examination is shorter than that of MRI, and the modalities have similar rates of sensitivity to detect lesions. CEM also costs less than MRI. ${ }^{(\mathbf{8})}$

Many studies have proved that CESM is superior to mammography in the identification of multiplicity, extent and size of malignant lesions especially in the dense breast parenchyma. ${ }^{(3)}$

Therefor the present study was conducted to investigate the potential benefits of using CESM in assessment of focal breast asymmetries detected on mammogram in 60 female patients over the course of one year.

\section{Subjects and Methods}

This study was case study \& carried out to investigate the potential benefits of using intravenous contrast material with full-field digital mammography in facilitation of characterization of focal breast asymmetry.
In this study, 60 persons are examined.

The Scientific Research Statistics Committee has approved the conduct of the research in 11/2020.

The study was carried out in national cancer institute, the institute and patients were had no problems with this study.

I carried out cases by myself. These cases are examined from 9/2019 to 3/2020.

\section{Statistical analysis}

The collected data were coded, entered, presented, and analyzed by computer using a data base software program, Statistical Package for Social Science (SPSS) version 22 Chicago. Ill. USA)

Qualitative data were represented as frequencies and percentage.

For quantitative variables mean, standard deviation (SD), and (minimum-maximum) were computed.

Chi square (X2) test was used to detect relation between different qualitative variables.

Sensitivity, specificity, predictive value for positive (PVP), predictive value for negative (PVN), and accuracy were calculated at $95 \%$ CI to measure the validity. 
The results were considered statistically significant and highly statistical significant when the significant probability ( $\mathrm{P}$ value) was $<0.05^{*}$ and $<0.001 * *$ respectively.

\section{Results:}

The mean age of included patients was $50.8 \pm 8.93$ years ranging from (37 to 74 ) years. About $66.7 \%$ of cases had lymph node enlargement. Only $6.7 \%$ of cases had nipple retraction. [Table 1]

CESM showed that two thirds of studied patients had mass enhanced lesions and the remaining one third had none mass enhanced lesions. About $50.0 \%$ of cases had micro-calcification with $6.7 \%$ of cases had parenchymal infiltration. About $63.3 \%$ had heterogeneous pattern of enhancement and $56.7 \%$ had intense degree. [Table 2]

As shown in table 3, the majority of lesions were malignant $(70.0 \%)$ with $71.4 \%$ of them were invasive duct carcinoma while benign lesions were only $30.0 \%$ with fibrocystic disease represented $44.4 \%$ of them.
Mammographic findings of mass lesions shows that $57.5 \%$ had micro calcification with $70.0 \%$ of cases had heterogeneous pattern of infiltration and $65.0 \%$ had intense degree of enhancement. [Table 4]

Regarding histopathological results of mass lesions, the majority of lesions were malignant $(82.5 \%)$ with $75.8 \%$ of them were invasive duct carcinoma while benign lesions were only $17.5 \%$ with fibrocystic disease and fibroadenoma represented $57.2 \%$ of them. [Table 5]

Table 6 shows that there was statistically significant difference $\left(\mathrm{p}<0.05^{*}\right)$ between malignant and benign cases regarding calcification and lymph nodes enlargement with $66.6 \%$ of malignant masses had microcalcification versus $14.3 \%$ of benign lesions and $84.9 \%$ of malignant cases had lymph node enlargement versus $28.6 \%$ of benign cases. 
Table (1): Demographic and clinical characteristics of the studied group ( $\mathrm{n}=60)$.

\begin{tabular}{|c|c|c|}
\hline Characteristics & Value & \\
\hline \multicolumn{3}{|l|}{ Age (years): } \\
\hline Mean \pm SD & $50.8 \pm 8.93$ & \\
\hline Range & $(37-74)$ & \\
\hline Items & No & $\%$ \\
\hline \multicolumn{3}{|l|}{ Side: } \\
\hline - Left & 29 & 48.3 \\
\hline • Right & 31 & 51.7 \\
\hline \multicolumn{3}{|l|}{ Lymph node involvement } \\
\hline$\bullet$ Yes & 40 & 66.7 \\
\hline$\bullet \quad$ No & 20 & 33.3 \\
\hline \multicolumn{3}{|l|}{ Lymph nodes } \\
\hline - Infra-mammary & 14 & 23.3 \\
\hline - Intra-mammary & 29 & 48.3 \\
\hline - Axillary & 4 & 6.7 \\
\hline - Supraclavicular & 3 & 5.0 \\
\hline • Subpectoral & & \\
\hline \multicolumn{3}{|l|}{ Nipple retraction } \\
\hline$\bullet$ Yes & 4 & 6.7 \\
\hline - $\quad$ No & 56 & 93.3 \\
\hline
\end{tabular}

Table (2): mammographic finding of the studied lesions ( $\mathrm{n}=60)$.

\begin{tabular}{lll}
\hline Variables & No. & $\%$ \\
\hline Mass lesions & $\mathbf{4 0}$ & $\mathbf{6 6 . 7}$ \\
Non mass lesions & $\mathbf{2 0}$ & $\mathbf{3 3 . 3}$ \\
Calcification: & & \\
No & $\mathbf{2 7}$ & $\mathbf{4 5 . 0}$ \\
Micro-calcification & $\mathbf{3 0}$ & $\mathbf{5 0 . 0}$ \\
Macro-calcification & $\mathbf{3}$ & $\mathbf{5 . 0}$ \\
Parenchyma infiltration & & \\
Yes & $\mathbf{4}$ & $\mathbf{6 . 7}$ \\
No & $\mathbf{5 6}$ & $\mathbf{9 3 . 3}$ \\
Enhancement & & \\
Homogenous & $\mathbf{2 1}$ & $\mathbf{3 5 . 0}$ \\
Heterogeneous & $\mathbf{3 8}$ & $\mathbf{6 3 . 3}$ \\
Ring & $\mathbf{1}$ & $\mathbf{1 . 7}$ \\
Degree of enhancement & & \\
Faint & 18 & 30.0 \\
Moderate & 8 & 13.3 \\
Intense & 34 & 56.7 \\
\hline
\end{tabular}


Benha medical journal, vol. 38, issue 2, 2021

Table (3): Histopathological results of the studied patients $(n=60)$.

\begin{tabular}{lll}
\hline Variables & No. & \% \\
\hline $\begin{array}{l}\text { Pathology results } \\
\text { Benign } \\
\text { Malignant }\end{array}$ & 18 & 30.0 \\
Pathological types of benign lesions: $(\mathbf{n = 1 8})$ & 42 & 70.0 \\
- Simple cyst & 1 & 5.6 \\
- Fibrocystic disease of the breast & 8 & 44.4 \\
- Fibroadenoma & 5 & 27.8 \\
- Abscess & 3 & 16.6 \\
- Granulomatous mastitis & 1 & 5.6 \\
Pathological types of malignant lesions $(\mathbf{n = 4 2})$ & & \\
$\bullet \quad$ Ductal carcinoma insitu & & \\
- Invasive duct carcinoma & 2 & 4.8 \\
- Invasive Lobular Carcinoma & 30 & 71.4 \\
\hline
\end{tabular}

Table (4): mammographic finding of mass lesions $(n=40)$.

\begin{tabular}{lll}
\hline Variables & No. & $\%$ \\
\hline Calcification: & 16 & 40.0 \\
No & 23 & 57.5 \\
Micro-calcification & 1 & 2.5 \\
Macro-calcification & & \\
Shape of the mass: & 9 & 22.5 \\
Rounded/oval & 31 & 77.5 \\
Irregular & & \\
Margin & 12 & 30.0 \\
Well defined & 28 & 70.0 \\
Ill defined & & \\
Parenchyma infiltration & 4 & 10.0 \\
Yes & 36 & 90.0 \\
No & & \\
Enhancement & 11 & 27.5 \\
Homogenous & 28 & 70.0 \\
Heterogenous & 1 & 2.5 \\
Ding & & \\
Faint & & 22.5 \\
moderate & 9 & 12.5 \\
Intense & 5 & 65.0 \\
\hline
\end{tabular}


Table (5): Histopathological results of the mass lesions $(n=40)$.

\begin{tabular}{lll}
\hline Variables & No. & $\%$ \\
\hline $\begin{array}{l}\text { Pathology results } \\
\text { Benign }\end{array}$ & $\mathbf{7}$ & $\mathbf{1 7 . 5}$ \\
Malignant & $\mathbf{3 3}$ & $\mathbf{8 2 . 5}$ \\
Pathological types of benign lesions: (n=7) & & \\
- Simple cyst & $\mathbf{1}$ & $\mathbf{1 4 . 3}$ \\
- Fibrocystic disease of the breast & $\mathbf{2}$ & $\mathbf{2 8 . 6}$ \\
- Fibroadenoma & $\mathbf{2}$ & $\mathbf{2 8 . 6}$ \\
- Abscess & $\mathbf{1}$ & $\mathbf{1 4 . 3}$ \\
- Granulomatous mastitis & $\mathbf{1}$ & $\mathbf{1 4 . 3}$ \\
Pathological types of malignant lesions (n=33) & & \\
- Ductal carcinoma insitu & & \\
- Invasive duct carcinoma & 2 & 6.1 \\
• Invasive Lobular Carcinoma & 6 & 75.8 \\
\hline
\end{tabular}

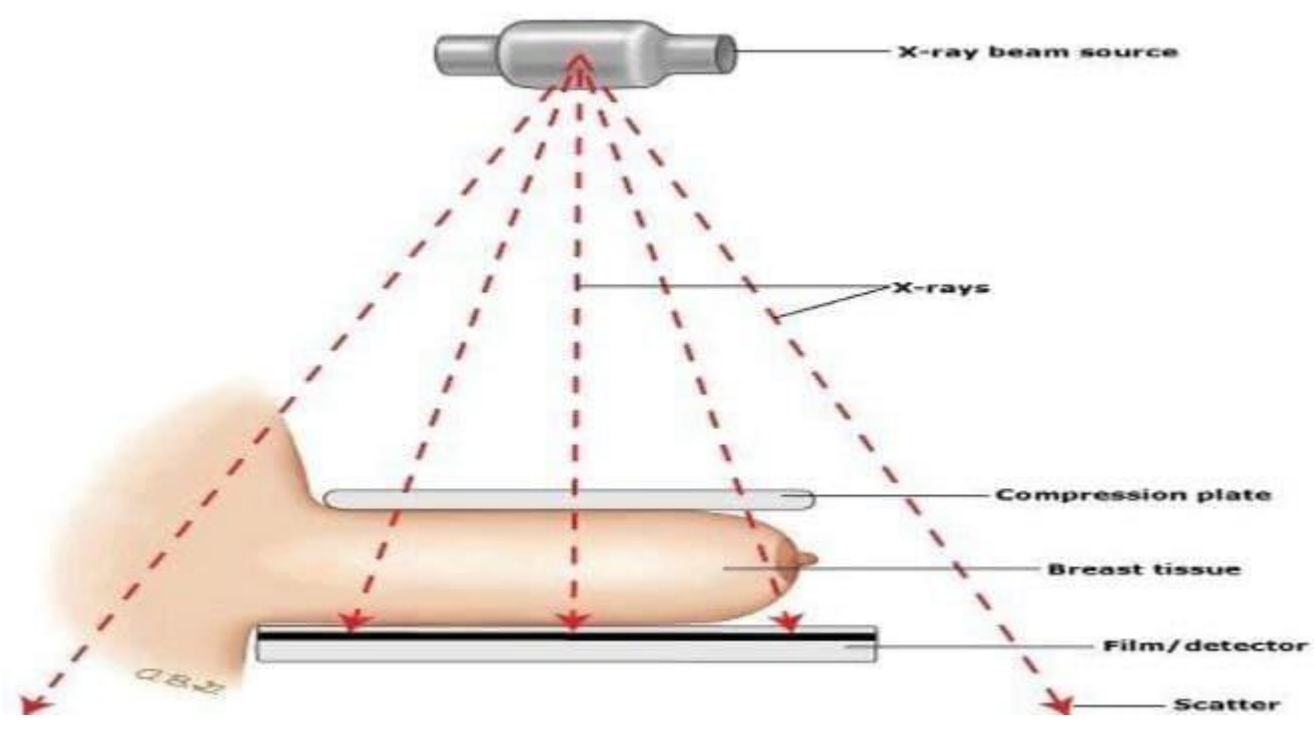

Fig. 1: Schematic diagram of a mammogram 
Table (6): Comparing clinical and radiological findings by contrast mammography between malignant and benign mass lesions $(\mathrm{n}=40)$

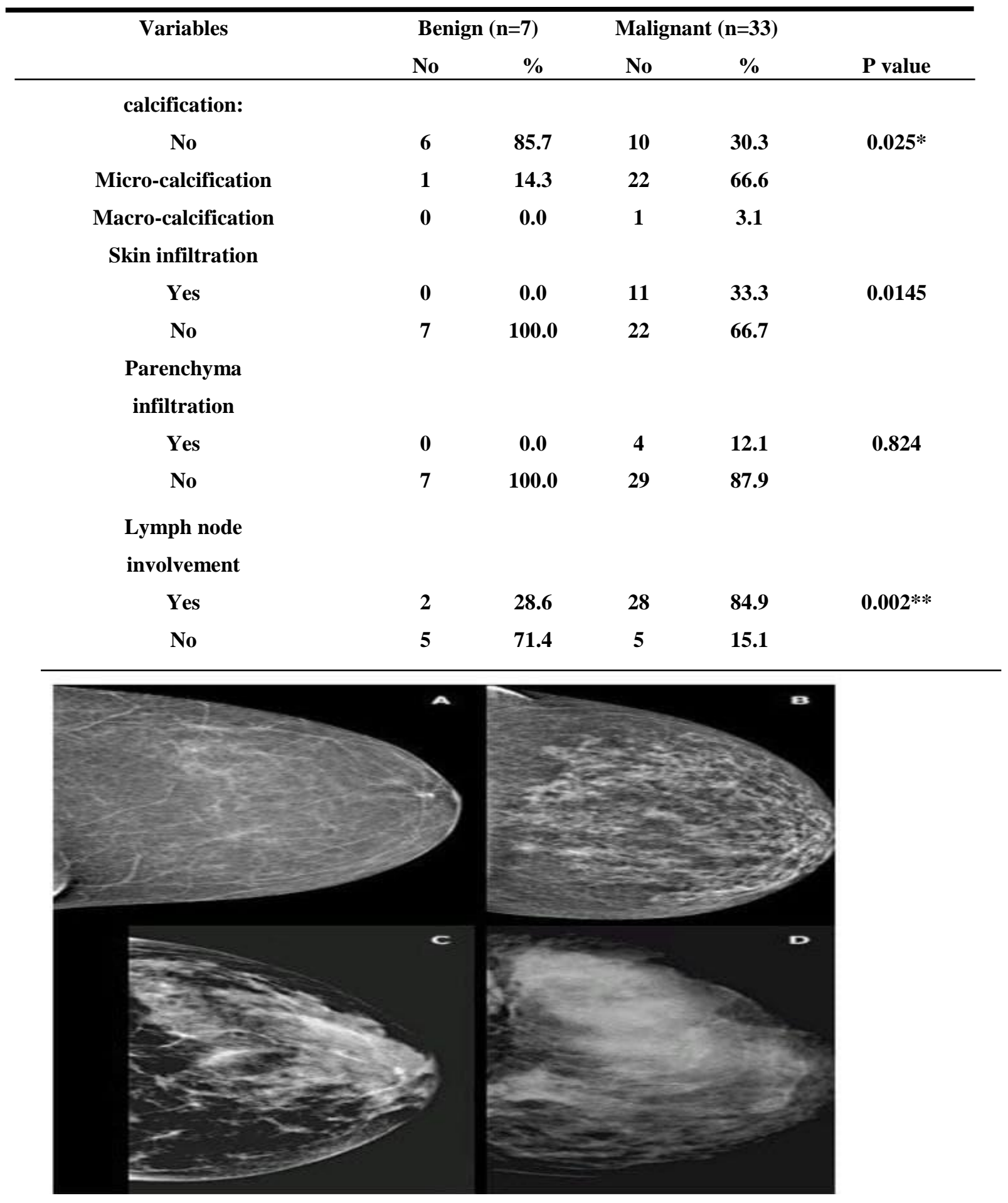

Fig. 2: Varying patterns of normal breast density using the standard BIRADS classification 


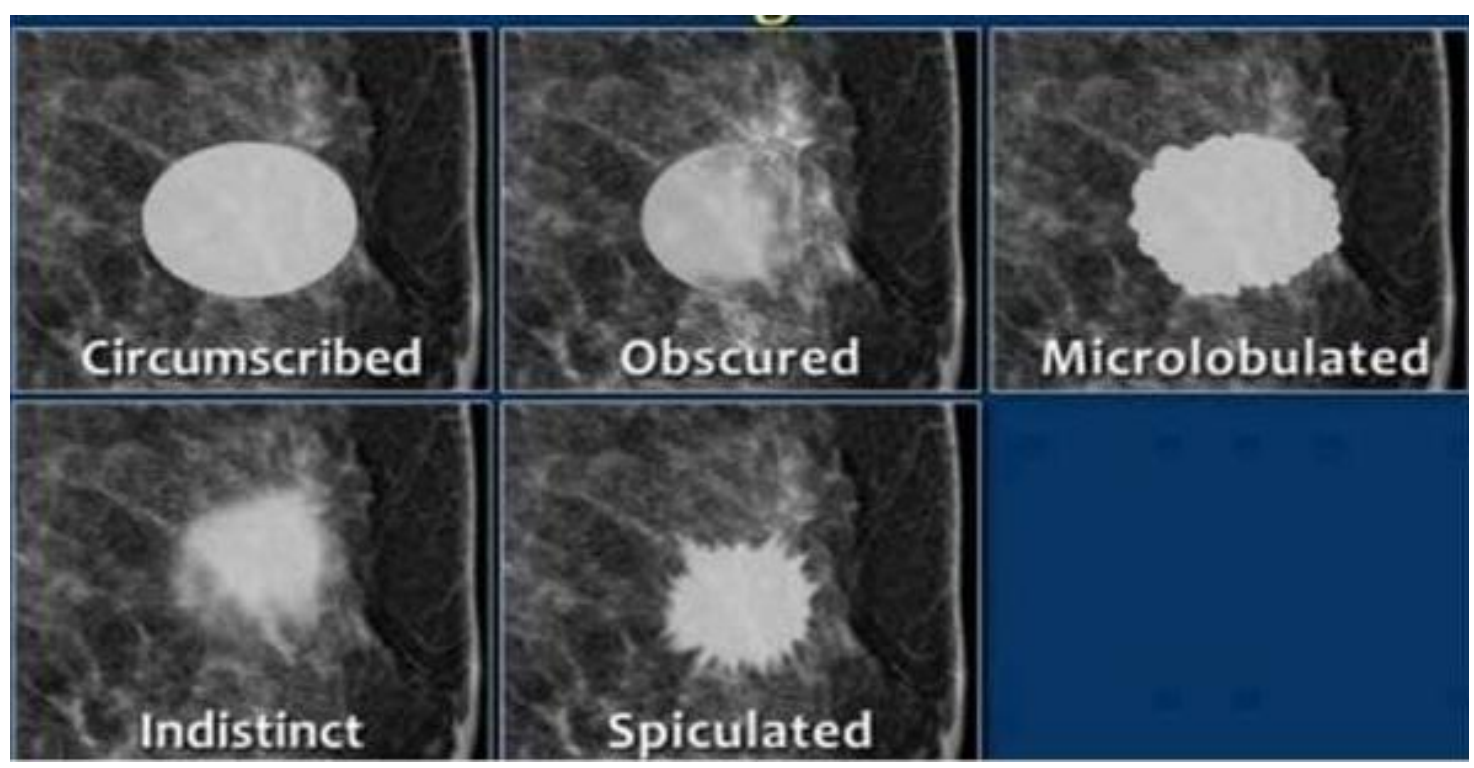

Fig.3: Mass margins (circumscribed, obscured, microlobulated, indistinct, and speculated). In general, these descriptors are arranged from least suspicious to most suggestive of Malignancy

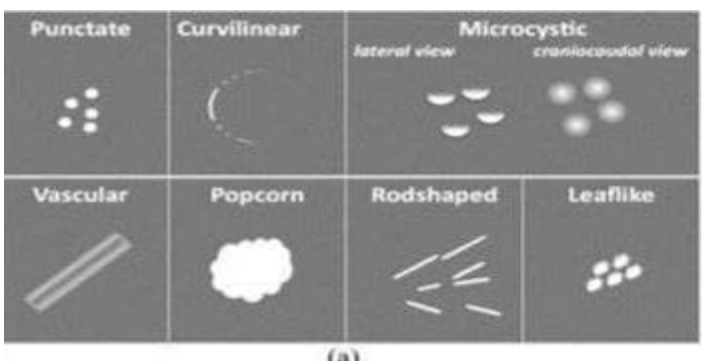

(a)

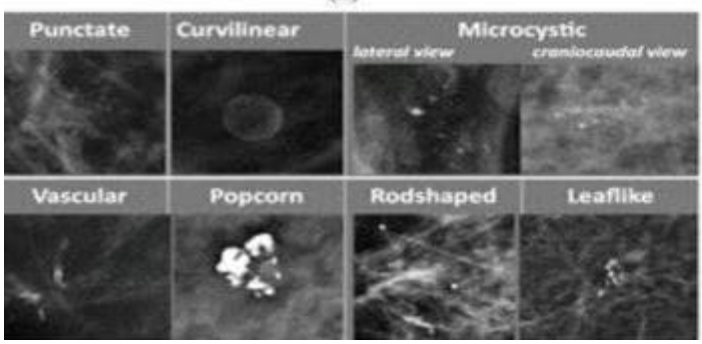

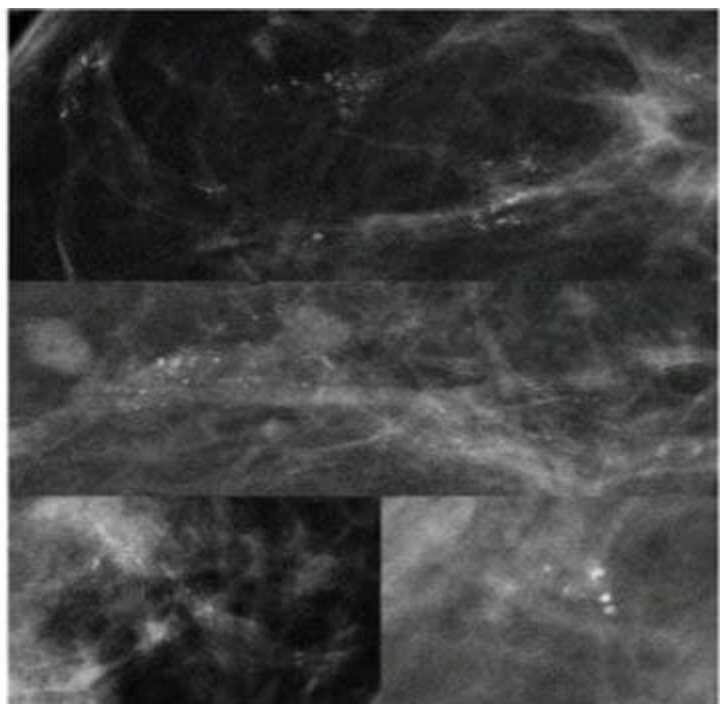

Fig.4: Patterns of calcification associated with a benign change; (b) examples of calcification associated with a benign change; and (c) examples of malignant calcification 


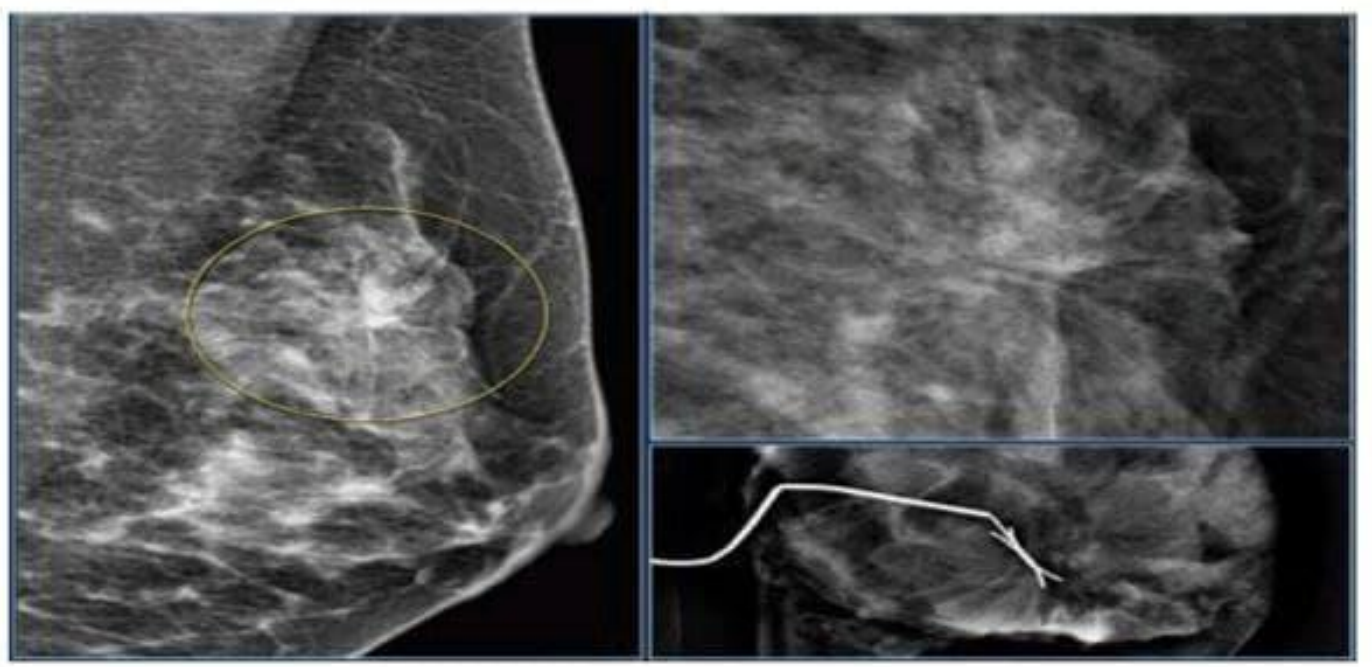

Fig. 5: Distortion of the normal breast architecture on oblique view (yellow circle) and magnification view

\section{Discussion}

CESM is a relatively new emerging imaging modality that provides better evaluation of breast masses by visualization of calcification depending on low-energy images combined with enhancement criteria on the contrast enhanced recombined images. ${ }^{(8)}$

Many studies reported that CESM is superior to mammography in the identification of multiplicity, extent and size of malignant lesions especially in the dense breast parenchyma. ${ }^{(3)}$

Therefor the present study was conducted to investigate the potential benefits of using CESM in assessment of focal breast asymmetries detected on mammogram in 60 female patients over the course of one year.

This study included 60 female patients with focal breast asymmetries detected on mammogram. Histopathology after ultrasound guided true cut biopsy was the standard reference for detecting lesions.

By the end of this study it was found that sensitivity of CESM 92.85\%, specificity $88.9 \%$ with accuracy $91.66 \%$ in assessment of detected focal asymmetry. In mass enhanced lesions shape of the mass had the highest sensitivity $90.0 \%$ and specificity $85.7 \%$ with accuracy $90.0 \%$. In non-mass lesions pattern of enhancement had the highest sensitivity $88.9 \%$. 
Regarding characteristics of studied participants, it was found that the mean age of included patients was 50.8 \pm 8.93 years ranging from (37 to 74) years. About $66.7 \%$ of cases had lymph node enlargement. Only $6.7 \%$ of cases had nipple retraction. CESM showed that two thirds of studied patients had mass enhanced lesions and the remaining one third had non mass enhanced lesions so all cases showed enhancement.

In agreement with an Egyptian study included 125 female patients had breast asymmetries on mammogram who presented for screening and diagnostic purposes, where the patients' mean ages were 48.87 years ranged from 25 to 81 years. Focal asymmetry was detected in $70.4 \%$ of cases and CESM showed that $56.8 \%$ of focal asymmetry cases had mass enhanced lesions, $34.1 \%$ had non-mass enhanced lesions and only 9\% showed non-enhanced lesions. ${ }^{(5)}$

Regarding a study aimed to assess the value of CESM in characterization of breast asymmetries and if it should be incorporated in its diagnostic work-up among 380 patients where focal asymmetry detected in $60 \%$ of them with mean age of 47 years ranged from 29 to 69 years. Associated mammography findings as edema, skin thickening, parenchymal distortion and calcifications were seen in $29 \%$ cases. ${ }^{(3)}$

The age of women with known breast carcinoma was 49.6 years ranged from 25 to 74 years. $^{(9)}$

Regarding histopathological results of studied patients, it was revealed that $70 \%$ of lesions were malignant with $71.4 \%$ of them were invasive duct carcinoma while benign lesions were only $30 \%$ with fibrocystic disease represented $44.4 \%$ of them. About three fourth of mass enhanced lesions were malignant $(82.5 \%)$. This indicated that focal asymmetries with enhancement are mainly in malignant lesions.

It is demonstrated that $26.1 \%$ of focal asymmetry cases were benign while $73.9 \%$ were malignant. Focal asymmetry showing mass enhancement was significantly correlated with malignancy, while nonenhancing focal asymmetry was correlated with benign pathology revealed that any enhancing asymmetry showing a mass or non-mass enhancement was significantly correlated with malignant pathology $(p \leq$ $0.001){ }^{(5)}$

This aimed to assess the value of CESM in characterization of breast asymmetries among 380 patients revealed that 
histopathology detected $23.2 \%$ benign lesions and $67.6 \%$ malignant lesions. ${ }^{(3)}$

It is showed that $57.4 \%$ of focal asymmetry cases were benign and $42.6 \%$ were malignant. Invasive duct carcinomas were the most encountered malignant tumour while adenosis and fibro-cystic changes were the most detected benign tumours. ${ }^{(10)}$

As regard morphological characteristics of enhanced malignant and benign lesions, in mass enhanced lesions there was highly statistically significant difference between benign and malignant lesions $(\mathrm{p}<0.001)$ regarding shape, margin, pattern and statistically significant $(\mathrm{p}<0.05)$ regarding degree of enhancement with the majority of malignant masses had irregular shape, illdefined margin, heterogeneous intense enhancement. In non-mass enhanced lesions there was statistically significant difference between benign and malignant lesions $(p<0.05)$ regarding enhancement pattern, degree of enhancement and distribution with the majority of malignant masses had heterogeneous intense enhancement with segmental distribution.

These results were supported by recent Egyptian study aimed to analyze the morphology and enhancement characteristics of breast lesions on CESM showes that in mass enhancement irregular mass shape, ill-defined or speculated margins, heterogeneous and intense internal enhancement all strongly correlated with a malignant pathology ( $\mathrm{p}$ value $\leq 0.001$ ). In non-mass enhancement intense and heterogeneous enhancement strongly correlated with a malignant pathology ( $p$ value $\leq 0.001)$. ${ }^{(10)}$

In consistence with presence of irregular shaped intensely enhancing mass lesions with ill-defined and speculated margins strongly correlated with malignant breast lesions. ${ }^{(11)}$

Regarding CESM findings and nature of lesions, it was found that there was highly statistically significant difference between benign and malignant breast lesions ( $p$ $<0.001)$ regarding pattern and degree of enhancement with the majority of malignant masses had heterogeneous intense enhancement in contrast to benign lesions with the majority had homogenous pattern of enhancement and faint degree.

It is demonstrated that heterogeneous pattern of contrast uptake was characteristic for malignant mass lesions ( $\mathrm{P} \leq 0.001)$. This could be explained by the fact that microvascular density plays a major role in 
determining the heterogeneity of tumor enhancement. ${ }^{(12)}$

In the present study, it was showed that there was statistically significant difference $(p<0.05)$ between malignant and benign cases regarding calcification and lymph nodes enlargement with $66.6 \%$ of malignant masses had microcalcification and $84.9 \%$ of malignant cases had lymph node enlargement.

It demonstrates that there was a significant correlation between focal asymmetry associated with distortion, suspicious calcification, skin/nipple changes and malignancy. Focal asymmetries with no other associated mammographic findings were significantly correlated with a benign pathology $(\mathrm{p} \leq 0.001){ }^{(5)}$

Regarding validity of CESM, it was found that CESM could predict 39 malignant lesions (true positive cases) out of 42 malignant lesions proven by histopathology with sensitivity $92.85 \%$ and can predict 16 benign lesions (true negative) out of 18 lesions proven by histopathology with specificity $88.9 \%$. PVP and PVN were $95.1 \%$ and $84.42 \%$ respectively with accuracy 91.66\%. CESM sensitivity increased to $93.9 \%$ in detection of mass enhanced lesions with accuracy92.5\%. In non-mass enhanced CESM sensitivity decreased to $88.9 \%$ with accuracy $90 \%$ while its specificity was $90.9 \%$.

In accordance with an Egyptian study to assess the value of CESM in characterization of breast asymmetries and if it should be incorporated in its diagnostic work-up found that CESM markedly improves the overall accuracy reaching $88.4 \%$ concluded that CESM is considered as a valuable complementary imaging tool considering the evaluation of breast asymmetries and should be incorporated in its diagnostic work-up in cases not resolved on an initial combined mammography and targeted ultrasound study especially in the presence of a heterogeneous dense breast parenchyma. ${ }^{(3)}$

CESM had a sensitivity of $89 \%$, a specificity of $89 \%$, PPV of $91 \%$, NPV of $86 \%$ and a diagnostic accuracy of $89 \%$. ${ }^{(13)}$

In an Egyptian study conducted to evaluate the clinical performance of CESM on asymmetries detected on a mammogram where $70.4 \%$ had focal asymmetry, CESM sensitivity, specificity, PVP and PVN were $100 \%, \quad 55.88 \%, \quad 85.85 \%$ and $100 \%$ respectively, with 15 false positive and no false negative findings concluded that focal asymmetries with other suspicious 
mammographic findings were statistically significant for malignancy and CESM played an important role in delineating tumor size and extension. ${ }^{(5)}$

In $10^{\text {th }}$ reference, it aimes to analyze the morphology and enhancement characteristics of breast lesions on CESM and to assess their impact on the differentiation between benign and malignant lesions demonstrated that CESM sensitivity was\%, specificity $83.3 \%$. PVP 85.8\%, PVN 93.4\% concluded that the assessment of the morphology and enhancement characteristics of breast lesions on CESM enhances the performance of digital mammography in the differentiation between benign and malignant breast lesions. ${ }^{(10)}$

CESM achieves sensitivity of $85 \%$, specificity of $89 \%$, PVP of $91 \%$, PVN of $86 \%$ and a diagnostic accuracy of $89 \%$. CESM showed slightly lower sensitivity and accuracy compared to MRI however because of being relative ease, available, cheap and acceptable by women, CEM can replace MRI as a problem-solving tool in the characterization of indeterminate breast lesions. ${ }^{(14)}$

The diagnoses based on CESM are slightly more reliable than those based on breast
MRI. The sensitivity of CESM examination was $100 \%$, higher than the $93 \%$ sensitivity of breast MRI ( $\mathrm{p} \leq 0.04)$. The accuracy of the CESM exam (79\%) was also higher than that of breast MRI (73\%) in their study, but this difference was not statistically significant. PVN was $100 \%$ for CESM and only $65 \%$ for breast MRI $(\mathrm{p}<0.001){ }^{(15)}$

It aims to determine feasibility of performing CESM and evaluate its performance compared with conventional digital mammography of histologically proven breast cancers as the gold standard which showed that the sensitivity increased to about $100 \%$ owing to CESM concluded that CESM was feasible and easily accomplished. It was used to detect known primary tumors at a rate comparable to that of MRI and higher than that of conventional digital mammography. ${ }^{(16,17,9)}$

Similarly, CESM, alone and in combination with mammogram, is as accurate as MRI but is superior to mammogram for lesion detection. Patients with dense breasts benefitted most from CESM with the smallest additional dose compared to mammogram. ${ }^{(18)}$

That conducts a comparative study between CESM and contrast enhanced MRI found that CESM showed slightly lower 
sensitivity $(88.89 \%)$ than breast MRI (96.3\%) however specificity was higher in CESM (66.67\%) than that of BMRI $(33.33 \%)$ concluded that in spite of the lower sensitivity of the CESM compared to MRI, the CESM appeared to be a suitable, easy, more comfortable, low cost and fast alternative to MRI in early detection of breast cancer recurrence specially for patients with contraindications to MRI. ${ }^{(19)}$ Similar observations were demonstrated that CESM sensitivity was $94.1 \%$ while MRI was $100 \%$ and CESM specificity $100 \%$ while MRI was $95.5 \%$. ${ }^{(20)}$

Finally, CESM is a developing modality used for the workup and management of breast cancer and has high sensitivity and specificity that used as complementary to standard mammogram and can replace breast MRI.

Regarding the validity of morphology descriptors for prediction of breast lesions on CESM, in mass enhanced lesions it was found that the shape of the mass had the highest sensitivity $90.0 \%$ and specificity $85.7 \%$ with accuracy $90.0 \%$. Enhancement pattern was $85.7 \%$ sensitive and $82.4 \%$ specific with the degree of enhancement had lesser sensitivity and specificity than the pattern. In non-mass enhanced lesions it was revealed that the pattern of enhancement had the highest sensitivity $88.9 \%$ while the pattern of distribution had the highest specificity $90.9 \%$ but the accuracy was highest regarding the pattern of enhancement $84.5 \%$

By comparing the morphology descriptors, in mass enhanced lesions the highest level of performance was scored by tumor margin description (sensitivity and PVP: 96.3\%, specificity and PVN: 83.3\%). (10) In nonmass enhanced lesions degree of enhancement and distribution had the highest sensitivity $86.4 \%$ while pattern of enhancement and its degree showed the highest specificity $(79.2 \%)$ concluded that the morphology descriptors of breast lesions on CESM are reliable in differentiating benign from malignant breast lesions with the exception of the ring pattern of contrast uptake.

\section{Conclusion}

CESM is valid and reliable tool in assessment of focal asymmetries. Enhancement and morphology characteristics are valuable in differentiation of benign and malignant breast lesions. It was found that sensitivity of CESM $92.85 \%$, specificity $88.9 \%$ with accuracy $91.66 \%$ in assessment of detected focal asymmetry. In 
mass enhanced lesions shape of the mass had the highest sensitivity $90.0 \%$ and specificity $85.7 \%$ with accuracy $90.0 \%$. In non-mass lesions pattern of enhancement had the highest sensitivity $88.9 \%$.

\section{References}

1) Mostafa AA, Eltomey MA, Elaggan AM, Hashish AA. Automated breast ultrasound (ABUS) as a screening tool: initial experience. Egyptian Journal of Radiology and Nuclear Medicine. 2019; 50(1):37.

2) Zhu X, Huang JM, Zhang K, Xia LJ, Feng L, Yang $\mathrm{P}$,et al . Diagnostic value of contrastenhanced spectral mammography for screening breast cancer: systematic review and metaanalysis. Clinical breast cancer. 2018; 18(5):e985-95.

3) Kamal RM, Moustafa AF, Fakhry S, Kamal EF, Radwan A, Hilal A, et al. Adding the merits of contrast to the ease of mammography; can we highlight what's behind breast asymmetries? Egyptian Journal of Radiology and Nuclear Medicine. 2019; 50(1):39.

4) Chesebro AL, Winkler NS, Birdwell RL, Giess CS. Developing asymmetries at mammography: a multimodality approach to assessment and management. Radiographics. 2016; 36(2):32234.

5) Wessam R, Gomaa MM, Fouad MA, Mokhtar SM, Tohamey YM. Added value of contrastenhanced mammography in assessment of breast asymmetries. The British journal of radiology. 2019; 92(1098):20180245.
6) Sickles EA, d'Orsi CJ, Bassett LW, Appleton CM, Berg WA, Burnside ES. Acr bi-rads® mammography. ACR BI-RADS® atlas, breast imaging reporting and data system. 2013; $5: 2013$.

7) Houben IP, Vanwetswinkel S, Kalia V, Thywissen T, Nelemans PJ, Heuts EM, et al. Contrast-enhanced spectral mammography in the evaluation of breast suspicious calcifications: diagnostic accuracy and impact on surgical management. Acta Radiologica. 2019; 60(9):1110-7.

8) Ghaderi KF, Phillips J, Perry H, Lotfi P, Mehta TS. Contrast-enhanced mammography: Current applications and future directions. RadioGraphics. 2019; 39(7):1907-20.

9) Jochelson MS, Dershaw DD, Sung JS, Heerdt AS, Thornton C, Moskowitz CS, et al. Bilateral contrast-enhanced dual-energy digital mammography: feasibility and comparison with conventional digital mammography and MR imaging in women with known breast carcinoma. Radiology. 2013; 266(3):743-51.

10) Kamal RM, Helal MH, Wessam R, Mansour SM, Godda I, Alieldin N. Contrast-enhanced spectral mammography: Impact of the qualitative morphology descriptors on the diagnosis of breast lesions. European journal of radiology. 2015; 84(6):1049-55.

11) Yoo JL, Woo OH, Kim YK, Cho KR, Yong HS, Seo BK, et al. Can MR imaging contribute in characterizing well-circumscribed breast carcinomas?. Radiographics. 2010; 30(6):1689704. 
12) Schnall MD, Blume J, Bluemke DA, DeAngelis GA, DeBruhl N, Harms S, et al. Diagnostic architectural and dynamic features at breast MR imaging: multicenter study. Radiology. 2006; 238(1):42-53.

13) Azzam H, Kamal RM, Hanafy MM, Youssef A, Hashem LM. Comparative study between contrast-enhanced mammography, tomosynthesis, and breast ultrasound as complementary techniques to mammography in dense breast parenchyma. Egyptian Journal of Radiology and Nuclear Medicine. 2020; 51(1):1-9.

14) Kamal RM, Hanafy MM, Mansour SM, Hassan M, Gomaa MM. Can contrast-enhanced mammography replace dynamic contrastenhanced MRI in the assessment of sonomammographic indeterminate breast lesions?. Egyptian Journal of Radiology and Nuclear Medicine. 2020; 51:1-8.

15) Łuczyńska E, Heinze-Paluchowska S, Hendrick E, Dyczek S, Ryś J, Herman K, et al. Comparison between breast MRI and contrastenhanced spectral mammography. Medical science monitor: international medical journal of experimental and clinical research; 21:1358.

16) Fallenberg EM, Dromain C, Diekmann F, Engelken F, Krohn M, Singh JM, et al. Contrast-enhanced spectral mammography versus MRI: initial results in the detection of breast cancer and assessment of tumour size. European radiology. 2014; 24(1):256-64.

17) Lobbes MB, Lalji U, Houwers J, Nijssen EC, Nelemans PJ, van Roozendaal $L$, et al. Contrast-enhanced spectral mammography in patients referred from the breast cancer screening programme. European radiology. 2014; 24(7):1668-76.

18) Fallenberg EM, Schmitzberger FF, Amer H, Ingold-Heppner B, Balleyguier C, Diekmann F, et al. Contrast-enhanced spectral mammography vs. mammography and MRIclinical performance in a multi-reader evaluation. European radiology. 2017; 27(7):2752-64.

19) Elfiky SM, Elsaid NA, Azeb EA, Elmor ME, Abdelaleem NA, Hassan TH. Comparison between the role of contrast enhanced mammography and dynamic contrast enhanced MRI in assessment of breast cancer recurrence. The Egyptian Journal of Hospital Medicine. 2018; 73(1):5875-85.

20) Yasin R, Abd El Ghany E. BIRADS 4 breast lesions: comparison of contrast-enhanced spectral mammography and contrast-enhanced MRI. Egyptian Journal of Radiology and Nuclear Medicine. 2019; 50(1):34.

To cite this article: Ghada S. Eliwa, Hesham E. Elshaikh, Gamal E. Elhabbaa, Lamia A. Salah Eldin Role of Contrast Enhanced Mammography in Assessment of Focal Breast Asymmetry. BMFJ 2021; 38(2): 640-656, DOI: 10.21608/bmfj.2021.60791.1378 